\title{
A Survey Comparing Adult and Child Psychiatry Trainees, Faculty, and Program Directors' Perspectives About Telepsychiatry: Implications for Clinical Care and Training
}

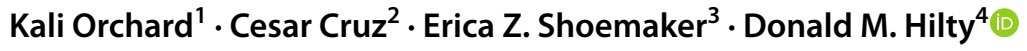

Received: 31 July 2020 / Revised: 21 November 2020 / Accepted: 2 December 2020 / Published online: 22 January 2021

(c) The Author(s), under exclusive licence to Springer Nature Switzerland AG part of Springer Nature 2021

\begin{abstract}
Telepsychiatry's effectiveness is well established, and interest in it is growing, despite few residency/fellowship core curricula and rotations. A link to a cross-sectional survey was sent via national organization listservs for psychiatry residents, fellows, faculty, and program directors to complete. The survey queried demographics, clinical experience, and views/concerns about telepsychiatry. Descriptive statistics and other analyses compared groups to assess the impact of amount clinical experience and psychiatric specialty (general vs. child and adolescent psychiatry), on interest, and views/concerns about the practice of telepsychiatry. All respondents $(N=270$; child psychiatry $N=89)$ have limited clinical experience with telepsychiatry $(46 \%$ overall; $49 \%$ of non-child had none versus $40 \%$ child). Trainees $(N=123$; child $N=43)$ expressed less interest than others. All respondents expressed worry about ability to do a physical exam, connectivity, medico-legal issues, and fit for diverse populations. Child respondents expressed less concern than others, but they reported more worry about loss of nonverbal cues. Clinical experience with telepsychiatry in the range of 6-20 $\mathrm{h}$ appears to build interest and allay concerns, though $1-5 \mathrm{~h}$ also may have a positive impact. More research is needed to assess clinical experience, interest, and concerns for adult and child psychiatry trainees and clinicians. Replicable, curricular interventions appear to be indicated.
\end{abstract}

Keywords Telepsychiatry $\cdot$ Telehealth $\cdot$ Telebehavioral $\cdot$ Child $\cdot$ Adolescent $\cdot$ Mental $\cdot$ Health $\cdot$ Psychiatry $\cdot$ Graduate . Medical · Education · Survey $\cdot$ Residents $\cdot$ Fellows $\cdot$ Concerns $\cdot$ Perceptions $\cdot$ Views

\section{Introduction}

Donald M. Hilty

donh032612@gmail.com

Kali Orchard

kali_orchard@hotmail.com

Cesar Cruz

cesarcruzmd10@gmail.com

Erica Z. Shoemaker

ezshoema@med.usc.edu

1 Yellowknife Adult and Child Psychiatry, Strongest Families Institute, Yellowknife, NT X1A 1L8, Canada

2 Child and Adolescent Psychiatry, USC Institute of Psychiatry \& Law, Torrance, CA 3848, USA

3 Department of Psychiatry \& Behavioral Sciences Keck School of Medicine, USC and LAC+USC Medical Center, LA, CA, USA

4 Department of Psychiatry \& Behavioral Sciences, University of California, 10535 Hospital Way, Mather, Davis, CA 95655, USA
Telepsychiatry (TP) or telebehavioral health (TBH) is effective for many types of psychiatric disorders, treatments, and populations (Hilty, Ferrer, Parish, Johnston, Callahan, \& Yellowlees, 2013; Gloff, LeNoue, Novins, \& Myers, 2015; Hubley, Lynch, Schneck, Thomas, \& Shore, 2016; Nelson \& Sharp, 2016). Initial concerns about therapeutic engagement, disconnections, and ability to simulate in-person care have subsided and outcomes are comparable (Hilty et al., 2013; Hubley et al., 2016) American Psychiatric Association (APA) and American Telemedicine Association (ATA), 2018). This pertains to care of child and adolescent outpatients (Gloff et al., 2015; Nelson \& Sharp, 2016; Hilty, Shoemaker, Myers, Snowdy, Yellowlees, \& Yager, 2016; American Academy of Child and Adolescent Psychiatry (AACAP) Committee on Telepsychiatry and AACAP Committee on Quality Issues, 2017; AACAP-APA, 2019; ATA, 2017), collaborative care in primary care (Myers, Vander Stoep, Zhou, McCarty, \& Katon, 2015; Hilty, 
Sunderji, Suo, Chan, \& McCarron, 2018a, b, c), and for diverse populations (Hilty, Feliberti, Evangelatos, Lu, \& Lim, 2018a, b, c) and patients in crisis or emergency settings (Reliford, \& Adebanjo, 2019). An e-behavioral health (e-BH) continuum has been described from persons/ patients using the Internet/web sites, chat groups, self-care management modules, social media, mobile health (e.g., apps, text), store-and-forward and video, or combinations thereof referred to as hybrid care (Hilty, Torous, Parish, Chan, Xiong, Scher, \& Yellowlees, 2020). Store-and-forward is defined as data collected (e.g., patient video interview, completion of questionnaire, diary) and then routed to the clinician for use at a later time.

Interest in technology by patients, medical students, and psychiatric trainees has significantly increased, initially fueled by video, web/Internet resources, and the electronic health record, then more recently by mobile health, social media and other technologies (Glover, Williams, Hazlett, \& Campbell, 2013; Teshima et al., 2016; Hilty, Torous, et al., 2020) Residents across medicine have attributed inadequate experience, false beliefs of teachers about telemedicine, and speculation about efficacy as the key limiting factors (Glover et al., 2013; Levy \& Strachan, 2013), and a survey of residents, program directors, and training directors found that experience is positively correlated with interest and negatively correlated with concerns (Cruz, Orchard, Shoemaker, \& Hilty, 2020). Technological expertise is variable, and early video systems had problems that negatively affected user experience. Despite these concerns, some patients, trainees and clinicians were hesitant to share their apprehensions (Hilty et al., 2013; Nelson, Cain, \& Sharp, 2017). Furthermore, patients, clinicians, and others adopt technology on a continuum from slowly to quickly based on motivation and other factors like generation/age (i.e., digital natives versus immigrants) (Wang, Myers, \& Sundaram, 2013). Ironically, as new generations adapt smartphones, social media, and other technologies, they have more personal technology experience than their teachers (Hilty, Chan, Torous, Luo, \& Boland, 2020). These mobile health technologies, particularly wearables with sensors, will transform child, adolescent, and family care by capturing events, symptoms, and ecologic data "in real time" for use in clinical decision making. These technologies may augment self-report and clinical assessments (Krishna, 2017; Odgers, \& Jensen, 2020; Russell, \& Gajos, 2020).

A broad call for more work in telepsychiatric education (Balon, Beresin, Coverdale, Louie, \& Roberts, 2015)— including an evidence-based pediatric telepsychiatry curriculum for trainees and practicing child and adolescent psychiatrists (Khan \& Ramtekkar, 2019)—stemmed from a significant gap in the literature (Sunderji, Crawford, \& Jovanovic, 2015; Crawford, Sunderji, López, \& Soklaridis, 2016). A survey of 46 programs revealed only 21 have a curriculum or informal clinical experience and 12 have only a curriculum (Hoffman \& Kane, (2015). Video competencies framed in the Accreditation Council for Graduate Medical Education Milestone domains (Hilty, Crawford, Teshima, Chan, Sunderji, Yellowlees, et al., \& Li, 2015; Hilty, Maheu, Drude, \& Hertlein, 2018a, b, c) was followed by social media (Zalpuri, Liu, Stubbe, Wrzosek, Sadhu, \& Hilty, 2018), telebehavioral health (Maheu et al., 2018; Maheu Drude, Hertlein, Lipschutz, Wall, Long, et al., \& Hilty, 2019), mobile health (Hilty, Chan et al., 2020), and asynchronous (Hilty, Torous et al., 2020) competency sets. Methods for teaching, evaluating, and administering them were included. Professionalism related to social media and medico-legal matters is a high priority (DeJong, 2018). It is unclear if learners (i.e., trainees) and those teaching them (i.e., clinicians/faculty) can keep up with the growing evidence base of technology clinical interventions and outcomes.

This study aimed the following:

1. Ask residents, fellows, program directors, and faculty about their clinical experience, interest in, and concerns about TP,

2. Compare child and adolescent psychiatry fellows, program directors, and faculty to those of other general residency and other fellowships in these areas,

3. Assess if and how clinical experience affects interest and concerns, so fellows are confident and have interest in pursuing TP in the future.

We hypothesized that parties' interest in TP and the type and degree of concerns may be based on clinical experience, and if experience was limited, it may affect their views or lead to misconceptions, which would be negatively correlated with interest and future practice with it.

\section{Methods}

This is an abbreviated summary of subjects, information collection and data, statistical analysis, and comparisons, as it is previously described (Cruz et al., 2020).

Subjects. The inclusion criteria were willingness to complete the survey and being a psychiatric resident, fellow, clinical faculty, or program director. The link to Survey Monkey was sent by e-mail to academic psychiatric organizations listservs (e.g., American Association for Directors of Psychiatry Residency Programs, Association For Academic Psychiatry) with a request to forward it to relevant parties; reminder emails were sent at 3- and 6-month follow-up. The only de facto exclusion criterion was not participating. There were no incentives or marketing materials.

Information collection (e.g., survey, other). Respondents were asked complete general information questions (e.g., 
geographic, professional demographics but not age, race or ethnicity), a Likert survey on TP clinical experience and interest, and a 47-item yes/no survey about views of TP; it took 10-15 min, was in English language, and was anonymous. Survey questions were iteratively developed, piloted, and revised before use (Cruz et al., 2020). The TP interest and clinical component used 5-item Likert like questions (e.g., for experience, options were no(ne), 1, 2-5, 6-20, $20+h)$

The 47-item true/false or yes/no survey was weighted equally with affirmative/negative questions used or adapted (Cruz et al., 2020) from 5 patient and clinician questionnaires (Horvath \& Greenberg, 1989; Stiles, Reynolds, Hardy, Rees, Barkham, \& Shapiro, 1994; Schneider, 1999; Yip, Chang, Chan, \& MacKenzie, 2003; Robillard \& Bouchard, 2004). The content of the questions were assorted, but subgrouping was done based on TP or TBH themes: clinical care effectiveness, specific patients/populations, communication, system/service issues, cost/reimbursement, legal, education/ training, and technical. Most questions fit patient care, systems-based practice, communication, practice-based learning, and knowledge Graduate Medical Education Milestone Competency domains. Internal and external consistency evaluation of the survey was not conducted.

Data, statistical analysis, and comparisons. Data received by the research team was kept in a password protected file. Before completing analyses, questionnaires were examined for completion; any missing items, the participant's responses on similar questions from the same category of the questionnaire were substituted (Downey \& King, 1998). Descriptive statistics (e.g., averages, percentages) and proportion of responses were calculated for each question. Cohorts were characterized by using proportions and means, if applicable. Between and intragroup comparisons (e.g., clinical experience with TP for Residents/Fellows [Rs/ Fs] versus Program Directors/ Faculty [PDs/Fac]; child Fs versus other Fs) were made based, when possible (i.e., if respondent numbers were not too low); a Pearson chi-square analysis was used for individual responses. Linear regression analysis, including multivariate regression analysis, was performed with participants' clinical experience as primary and interest as secondary factors, then another analysis in reverse order. For all analyses, a significance level of 0.05 , which corresponded to a confidence interval of $95 \%$, was used to determine statistical significance.

Institutional review board (IRB). An IRB exemption was granted in 2017, as this was an anonymous pre-COVID-19 survey.

\section{Results}

\section{All Respondent Data $(N=270)$}

Subjects totaled 270, with 76 Rs, 47 Fs, 57 PDs, and 90 Fac. Most Rs/Fs responders were from general psychiatry (54\%), child and adolescent (33\%), and other fellowships (13\%; forensic, geriatric, psychosomatic, and substance). In terms of geography for all responders, $76 \%$ were practicing in an urban setting.

Clinical experience with TP varied between all and Rs/ Fs respondents, respectively, from none ( 46 to $54 \%$ ), $1 \mathrm{~h}$ (11 to $14 \%$ ), $2-5 \mathrm{~h}$ (13 to $9 \%$ ), $6-20 \mathrm{~h}$ (9 to $12 \%$ ), or $20+\mathrm{h}(21$ to $11 \%)$. In terms of interest in TP, overall, $68 \%$ of respondents were interested or very interested, $22 \%$ undecided, $7 \%$ uninterested, and $4 \%$ very uninterested; Rs/ Fs had less interest, with $58 \%$ interested or very interested, $26 \%$ undecided, $11 \%$ uninterested, and $6 \%$ very uninterested.

The 10 most common concerns about TP for all, Rs/ Fs, and PDs/Fac, respectively (Table 1), were as follows: that one cannot perform a physical exam $(54 \%, 64 \%, 47 \%)$,

Table 1 Top 10 concerns about telepsychiatry: a comparison of all respondents, residents/fellows, and program directors/faculty

\begin{tabular}{|c|c|c|c|c|}
\hline \# & Item/Concern & All $N=270$ & $\mathrm{R} / \mathrm{F} N=123$ & $\mathrm{PD} / \mathrm{Fac} N=147$ \\
\hline 1 & One cannot perform a physical exam & $54.47 \%$ & $63.64 \% *$ & $47.06 \%$ \\
\hline 2 & Poor internet connection is a roadblock to implementing TP & $51.63 \%$ & $54.55 \%$ & $49.26 \%$ \\
\hline 3 & Liability risks involved with TP are unknown & $46.75 \%$ & $50.91 \%$ & $43.38 \%$ \\
\hline 4 & Certain cultures will be less accepting & $39.62 \%$ & $46.36 \% *$ & $33.09 \%$ \\
\hline 5 & Non-verbal cues will be missed & $35.77 \%$ & $44.55 \% *$ & $28.68 \%$ \\
\hline 6 & Privacy is an issue & $32.52 \%$ & $35.25 \%$ & $30.15 \%$ \\
\hline 7 & $\mathrm{TP}$ is not as effective as to in-person psychiatry & $32.11 \%$ & $37.27 \%$ & $27.94 \%$ \\
\hline 8 & One cannot manage emergencies related to safety with TP & $29.67 \%$ & $49.09 \% *$ & $22.06 \%$ \\
\hline 9 & Residency is insufficient for one to become competent in TP & $29.67 \%$ & $40.91 \% *$ & $20.59 \%$ \\
\hline 10 & Paranoid patients do not like TP & $26.42 \%$ & $42.67 \% *$ & $13.24 \%$ \\
\hline
\end{tabular}

$F a c$ faculty, $F$ fellow, $P D$ program director, $R$ resident, $T P$ telepsychiatry

* Significant with $p<0.05$ in comparing Rs/Fs versus PDs/Fac 
poor Internet connection is a roadblock to implementing TP $(52 \%, 55 \%, 49 \%)$, liability risks involved with TP are unknown $(47 \%, 51 \%, 43 \%)$, and perceptions or misperceptions that patients from certain cultures will be less accepting $(39 \%, 46 \%, 33 \%)$; the literature shows all cultures receive TP well (Hilty, Ferrer et al., 2013). Rs/ Fs were significantly more concerned about handling emergencies related to safety with TP than the PDs/Fac (49 to $22 \%$ ), as well as about residency being insufficient to become competent in TP (41 to $21 \%$ ).

\section{Impact of Clinical Experience on Interest}

Respondents with none or only one encounter of TP reported being very interested (25\%), interested (33\%), undecided $(25 \%)$, uninterested $(10 \%)$, and very uninterested $(5 \%)$. As clinical experience increased from none, to $1-5 \mathrm{~h}$ and to $6-20 \mathrm{~h}$, interest in TP increased from 58 to $74 \%$ to $82 \%$ (Fig. 2).

\section{Impact of Clinical Experience on Views}

Those with $0-5 \mathrm{~h}$ of clinical experience had statistically higher concerns than those with $6-20 \mathrm{~h}$, respectively: one cannot perform a physical exam (59 to $42 \%$ ), liability risks (54 to $28 \%$ ), poor Internet as a road block (54 to $36 \%$ ), certain cultures are less accepting ( 42 to $31 \%$ ), and nonverbal cues are missed with TP (41 to $24 \%$ ).

\section{Impact of Interest on Views}

Interest was high—with groups interested or very interested in TP (66\%)-regardless of clinical experience: $0-1 \mathrm{~h}$ (42\%), $1-5 \mathrm{~h}(11 \%), 6-20 \mathrm{~h}(10 \%)$, or $20+\mathrm{h}(27 \%)$. Their concerns were similar to the overall group, as were those of the undecided group.

\section{Residents/Fellows (Rs/Fs) Data $(N=123)$}

\section{Overview}

Rs/Fs were numerically, but not statistically, less interested in TP than all participants or, more specifically, PDs/Fac. Respondents typically practice in an urban setting (81\%) have clinical experience with TP (54\%) and have significant interest in TP $(66 \%)$ (interested or very interested).

\section{Impact of Clinical Experience on Interest and Views/ Concerns}

Of the group with $0-5 \mathrm{~h}$ of clinical experience, $66 \%$ were noted to be interested or very interested, $24 \%$ were undecided, and only $8 \%$ were uninterested or very uninterested. As clinical experience increased, those very interested or interested increased: none (58\%), $1-5 \mathrm{~h}(61 \%)$, $6-20 \mathrm{~h}(78 \%)$, and $20+\mathrm{h}(83 \%)$ - the shift from $1-5$ to $6-20 \mathrm{~h}$ was substantial.

Subgroup analyses of respondents with $0-5 \mathrm{~h}$ versus $6+\mathrm{h}$ both groups were concerned about poor Internet connection as is a roadblock to good care and about ability to perform a physical exam. The $6+\mathrm{h}$ group, though, had statistically less concerns that nonverbal cues are missed $(23 \%)$ and that paranoid patient do not like TP $(19 \%)$.

\section{Impact of Interest on Views/Concerns}

Those with high interest in TP had variable clinical experience with TP, and their concerns did not statistically differ from all residents or all respondents. Approximately $80 \%$ of those uninterested or very uninterested had no clinical experience, and their concerns were statistically significant versus all respondents and Rs/Fs in terms of loss of nonverbal cues $(67 \%)$, ineffectiveness $(50 \%)$, poor Internet connection (50\%), and TP as ineffective (50\%). Those undecided in terms of interest did not vary from the other groups in experience or concerns.

\section{Program Directors/Faculty (PDs/Fac) Data $(N=147)$}

\section{Overview}

PDs/Fac had no (39\%), one encounter to $5 \mathrm{~h}(23 \%), 6-20$ $(8 \%)$, or $20+\mathrm{h}(30 \%)$ of clinical experience with TP.

\section{Impact of Clinical Experience on Concerns}

Of the 90 respondents with $5 \mathrm{~h}$ or less clinical experience with TP, $57 \%$ of these responders reported they are interested or very interested in TP, while $25 \%$ were undecided, and $16 \%$ uninterested or very uninterested. Their concerns did not vary compared with all respondents or to other PDs/Fac or Rs/Fs, specifically. 
A COMPARISON OF CLINICAL EXPERIENCE IN

TELEPSYCHIATRY FOR ALL PSYCHIATRISTS (N=270), ADULT

( $\mathrm{N}=181$ ) AND CHILD ( $\mathrm{N}=\mathbf{8 1}$ ) PSYCHIATRISTS (in \%)

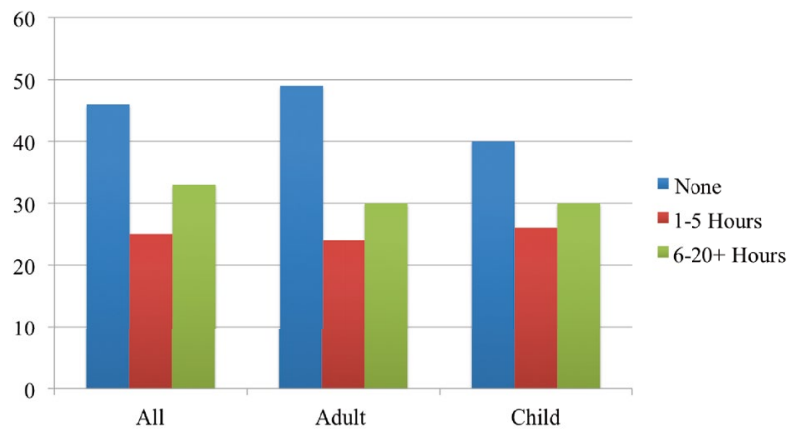

Fig. 1 A comparison of clinical experience in telepsychiatry for all psychiatrists $(N=270)$, adult $(N=181)$, and child $(N=81)$ psychiatrists (In \%)

\section{Impact of Interest on Concerns}

A high percentage (79\%) PDs/Fac were noted to be interested to very interested in TP. Their clinical experience varied: $30 \%$ none, $10 \%$ a one-time encounter, $13 \%$ up to $5 \mathrm{~h}, 9 \%$ from 6 to $20 \mathrm{~h}$, and $38 \%$ with more than $20 \mathrm{~h}$ of TP. They had less concern about liability risk (36\%), though not significant, and rated items like "primary provider may not follow through on recommendations made by the telepsychiatrist" (32\%) and "TP offers poor reimbursement" $(30 \%)$ higher than others.

The undecided group varied in clinical experience-53\% had no clinical experience, $19 \%$ had up to $5 \mathrm{~h}, 9 \%$ had $6-20 \mathrm{~h}$, and $19 \%$ had $20+\mathrm{h}$ of TPand their concerns did not differ from other groups

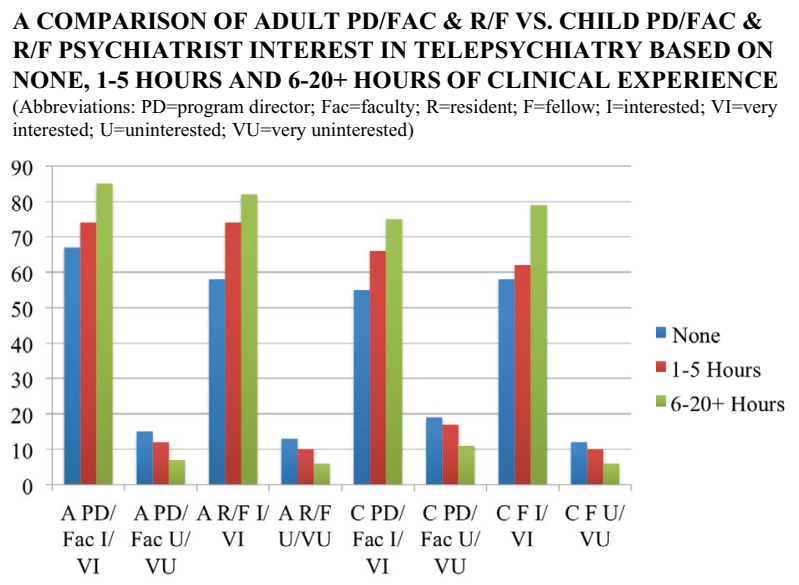

Fig. 2 A comparison of adult program director/faculty and resident/ fellow vs. child program director/faculty and resident/fellow psychiatrist interest in telepsychiatry based on none, $1-5 \mathrm{~h}$ and $6-$ $20+\mathrm{h}$ of clinical experience except for much less high interest (30\% to 53\%). The not interested group also had much less clinical experience $(63 \%)$ and much higher concerns that nonverbal cues are missed $(73 \%)$ and that TP is not as effective as in-person psychiatry $(60 \%)$.

\section{Comparing Child Psychiatrists to Non-child Psychiatrists $(N=89)$}

\section{Overview}

Child psychiatry respondents included $39 \mathrm{Fs}, 30 \mathrm{Fac}, 17$ PDs, and 3 Rs; practice geography did not differ. Child psychiatrists' clinical experience with TP, compared with all respondents and adult respondents, respectively, varied from none ( $40 \%$ vs. $46 \%$ vs. $49 \%$ ), $1-5$ (26\%, vs. $24 \%$ vs. $25 \%)$, and $6-20+(34 \%$ vs. $30 \%$ vs. $33 \%)$ h (Fig. 1 ). This shows the adult psychiatry group had more with none (red) or $1-5$ (blue) h, the child psychiatry group has more experience at $6-20+$ (green) hours, and there are statistically fewer child psychiatrists with no experience (40\%) than others (49\%). The most common child PDs/Fac replies, compared with the non-child ones, were no/none (41 to $40 \%), 1-5 \mathrm{~h}$ (26 to $20 \%), 6-20 \mathrm{~h}$ (17 to $12 \%)$, and $20+\mathrm{h}$ (16 to $28 \%)$ of TP. The most common child Fs replies, compared with the non-child ones, were no/none (46 to $58 \%), 1-5 \mathrm{~h}$ (22 to $24 \%), 6-20 \mathrm{~h}$ (22 to $13 \%$ ), and $20+\mathrm{h}(10$ to $5 \%)$ of TP; the group with no experience was significantly less than for other respondents.

Interest in TP for child psychiatrists varied from very interested $(32 \%)$, interested $(33 \%)$, undecided $(25 \%)$, uninterested $(9 \%)$ to very uninterested $(1 \%)$ technically more interest, but not statistically different from all respondents. Overall, respondents for child Fs, PDs, and Fac, compared with non-child ones, revealed little difference in the interested or very interested (65 to $68 \%$ ), undecided ( 25 to $21 \%$ ), or not interested or very uninterested (10 to $11 \%$ ) groups.

Adult PDs/Fac were more interested than adult R/Fs, child PDs/Fac, and child Fs regardless of TP exposure (i.e., none, $1-5 \mathrm{~h}$ and $6-20+\mathrm{h}$ ) (Fig. 2). A shift upward in interest for some (e.g., adult Rs/Fs) was noticed between none and $1-5 \mathrm{~h}$ of exposure, while the shift for others was with $6-20+$ h (e.g., child Fs). All groups trended toward more interest with increased exposure. Child PDs/Fac were equally interested/very interested and uninterested/very uninterested as child Fs.

Child psychiatry respondents appeared to have less serious concerns, compared with non-child ones (Table 2), with statistically lower differences in inability to perform a physical exam (40 to 60\%); poor Internet connection is a roadblock to TP (41\% to $57 \%)$ and liability risk ( $40 \%$ to 
Table 2 A comparison of child and non-child psychiatrist' top 10 concerns about telepsychiatry

\begin{tabular}{lll}
\hline Item/Concern & Child & Non-child \\
\hline One cannot perform a physical exam with TP & $43.21 \%$ & $60.00 \% *$ \\
Nonverbal cues are missed with TP & $42.98 \%$ & $32.73 \% *$ \\
Poor internet connection is a roadblock to TP & $40.74 \%$ & $56.97 \% *$ \\
The liability risks involved in TP are unknown & $39.51 \%$ & $50.30 \%^{*}$ \\
Certain cultures will be less accepting of TP & $38.27 \%$ & $39.39 \%$ \\
One cannot manage emergencies related to safety with TP & $30.88 \%$ & $29.09 \%$ \\
TP is not as effective as face-to-face psychiatry & $28.40 \%$ & $33.94 \%$ \\
Clinicians find TP to be too impersonal & $28.40 \%$ & $24.24 \%$ \\
Residency training is insufficient for one to become competent with TP & $27.16 \%$ & $30.91 \%$ \\
Disruptive behavior cannot be managed with TP & $25.93 \%$ & $26.67 \%$ \\
\hline
\end{tabular}

$T P$ telepsychiatry

*Significant with $p<0.05$ in comparing child to non-child respondents
$50 \%)$. An exception to that was a greater concern about loss of non-verbal cues, which was statistically higher for child psychiatrists than non-child psychiatrists (42 to 33\%).

\section{Impact of Clinical Experience on Views/concerns}

Child psychiatrists with no experience $(N=36)$ worry more than those with $20+\mathrm{h}$. Of those with no experience with TP, $40 \%$ or more endorsed concern about 9 of the 10 items presented (Table 3). The highest areas of concern were liability risk $(60 \%)$, inadequate TP training in residency $(46 \%)$, missing non-verbal cues $(43 \%)$, and inability to manage emergencies related to safety (40\%). Other groups $(1,2-5,6-20$, and $20+\mathrm{h}$ paralleled nonchild psychiatry counterparts, if not already specifically discussed, for both Rs/Fs and all respondents (i.e., fewer concerns with $2-5 \mathrm{~h}$ and much fewer with $6-20 \mathrm{~h}$ of clinical experience).

\section{Impact of Clinical Experience on Interest}

Similar to the experience of Rs/Fs, overall, of the child psychiatry respondents with no exposure to TP at all $(N=36), 22 \%$ are very interested, $36 \%$ are interested, $33 \%$ undecided, $6 \%$ uninterested, and $3 \%$ very uninterested. As clinical experience increased from none, to $1-5 \mathrm{~h}$ and to $6-20 \mathrm{~h}$, interest in TP increased from 58 to $66 \%$ to $79 \%$; adult Rs/Fs were similar at 58 to $74 \%$ to $82 \%$ (Fig. 2). Those uninterested and very interested ranged from 12 to $10 \%$ to $6 \%$, compared with adult Rs/Fs from 13 to $8 \%$ to $6 \%$ (Fig. 2).
Table 3 A comparison of concerns about telepsychiatry between child psychiatrists with no experience versus compared with those with multiple encounters

\begin{tabular}{lll}
\hline Item/Concern & $\begin{array}{l}\text { No experience } \\
N=36^{1}\end{array}$ & $\begin{array}{l}\text { Multiple } \\
\text { encoun- } \\
\text { ters } N=22^{1}\end{array}$ \\
\hline The liability risks involved in TP are unknown & $60.00 \%$ & $18.18 \%^{*}$ \\
Residency training is insufficient for one to become competent with & $45.71 \%$ & $4.54 \%^{*}$ \\
TP & $42.86 \%$ & $40.91 \%$ \\
Nonverbal cues are missed with TP & $42.86 \%$ & $40.91 \%$ \\
One cannot perform a physical exam with TP & $40.00 \%$ & $18.18 \% *$ \\
One cannot manage emergencies related to safety with TP & $40.00 \%$ & $13.64 \%^{*}$ \\
TP is not as effective as face-to-face psychiatry & $40.00 \%$ & $45.45 \%$ \\
Poor internet connection is a roadblock to TP & $40.00 \%$ & $13.64 \%$ \\
Clinicians find TP to be too impersonal & $40.00 \%$ & $31.82 \%$ \\
Certain cultures will be less accepting of TP & $37.14 \%$ & $18.18 \% *$ \\
Paranoid patients do not like TP & & \\
\hline
\end{tabular}

$T P$ telepsychiatry ${ }^{1}$ Overall child psychiatry $N=89$; this is a comparison of two subgroups only

*Significant with $p<0.05$ in comparing no with significant experience (i.e., multiple encounters) 


\section{Discussion}

There are several preliminary findings of this survey of clinical experience, interest, and views/concerns, particularly for child and adolescent psychiatry. First, child psychiatrists have a little more clinical experience than other respondents, but it is probably still limited due to time, competing interests and number of TP or TBH options. Second, all respondents' interest in TP is high - consistent with other surveys (Glover et al., 2013; Sunderji et al., 2015)—and child psychiatry interests appears a little higher than that of nonchild respondents (Cruz et al., 2020). There may be many patient, clinician, and system reasons for this, aside from effectiveness, evidence base, and therapeutic engagement (Hilty et al., 2013; Gloff et al., 2015; Hubley et al., 2016; Nelson \& Sharp, 2016; Hilty, Randhawa, Maheu, McKean, Pantera, \& Rizzo, 2020a, b, c, d). It may not just be a generational issue related to technology adoption data and assumptions (Wang et al., 2013), as non-child Rs/Fs have less interest than PDs/Fac. Fellows work with children who have grown up around technology and are comfortable seeing their doctor by video, with some teenagers and those with autism preferring it (Pakyurek, Yellowlees, \& Hilty, 2010). TP limits unnecessary travel, reaches rural populations, and reduces school absences and parental time off from work (Gloff et al., 2015). It can also streamline clinical workflows, enable continuity of care, facilitate collaboration among virtual care teams, and improve flexibility in work schedules (Gloff et al., 2015; Khan \& Ramtekkar, 2019). For physicians, this may enhance physician satisfaction and well-being, while reducing isolation.

It is premature to conclude that a lack of exposure is negatively impacts trainee interest - though clinical experience appears to predict interest-but lack of exposure directly leads to inadequate knowledge and concerns which may or may not be well founded. Fortunately, child psychiatrists appear to have fewer and less strongly held concerns than non-child respondents, and remarkably, the intensity (i.e., percentages) of concerns drops with $1,2-5$, and $6-20 \mathrm{~h}$ of clinical experience. The study was not geared to measure the "dose" needed to feel comfortable with TP, but data suggest that a threshold of $5-6 \mathrm{~h}$ may debunk preliminary questions/concerns and $6-10+\mathrm{h}$ may work to develop skills; what residents versus fellows need may be similar or different, as well as based on other factors. This is important, as those physicians that are undecided, uninterested, and very uninterested have higher percentages of concerns, which will help these individuals opt out if they do not find technology as a good fit individually. PDs/Fac cannot depend on interest in TP to develop into a high level of expertise. Attitude (e.g., interest) is not a substitute for supervised clinical experience to develop knowledge and skill (Pratt, 1998). Regardless, child psychiatry respondents express fewer concerns. This may imply a flexibility and adaptability, perhaps due to working in/with a variety of settings/populations.

The concerns of child psychiatrists have important implications for clinical care and training. The child and adolescent psychiatry respondents were overall less concerned than other respondents about the potential negative impact of TP, such as an inability to perform a physical exam, loss of nonverbal cues, potentially poor fit with culturally diverse populations, managing emergencies, dealing with patients' paranoia, general effectiveness, connectivity, and medico-legal issues (e.g., liability) (Cruz et al., 2020). Pediatric emergency TP is rapidly being established (Reliford \& Adebanjo, 2019). Flexibility, adaptability, and interprofessional teamwork will help with performing a physical exam, and similar support or other interventions may be needed to detect nonverbal cues. These are relied on quite heavily, as children frequently are not able to communicate their experiences with words (Nelson et al., 2017; Odgers \& Jensen, 2020). The challenges of telepsychiatry may still pale in comparison to challenges and concerns about social media (e.g., cyberbullying, engagement) (Zalpuri et al., 2018), mobile health (e.g., privacy, boundaries, integration of workflow) (Hilty, Chan et al., 2020), and other asynchronous technologies (e.g., addictions or problematic use) (Hilty, Torous et al., 2020; Joshi, Stubbe, Li, \& Hilty, 2019).

There is still substantial clinical, educational, research, and faculty development needed related to technology. Most participants in health care, and particularly those working in health systems, are moving forward with technology based on patient needs and the evidence base - aside from the external mandate to "flatten the curve" of COVID-19 spread and "accelerate and bend the curve" of digital health care (Torous, Jän Myrick, Rauseo-Ricupero, \& Firth, 2020)—which will "dose" trainees and clinicians alike with clinical experience in TP. Effectiveness and implementation research is needed to help PDs find the curricular dose for TP, though this may depend on general residency training, which could intervene earlier with tangible clinical experiences that may improve Rs/ Fs' confidence for future TP practice, and serve as a foundation for subspecialty training, in general. A model of core residency and augmented child TP training has heuristic value, but PDs may be more inclined to design/obtain rotations for electives rather than required rotations due to time constraints, faculty expertise and other factors. As many training programs may struggle to amass the resources required to meet the suggested competency requirements for video, social media, mobile health, and asynchronous technologies in combination with cultural competencies (Hilty, Gentry, McKean, Cowan, Lim, \& Lu, 2020; Hilty, Crawford et al., 2015; Hilty, Maheu et al., 2018a, b, c; Zalpuri et al., 2018), a strong case can be made 
for an inter-institution pooling of resources (i.e., curricula, faculty teachers/supervisors, evaluation processes). Institutional competencies have been suggested, which would consolidate clinical care, residency/fellow training, and faculty development missions around core competencies (Hilty, Torous et al., 2020; Hilty, Unützer, Ko, Luo, Worley, \& Yager, 2019). Faculty that have not trained in the use of technology in clinical settings would benefit from the training the fellows receive to ensure that they adapt, remain current in skills, and are poised to effectively supervise (Armstrong, Mackey, \& Spear, 2004).

There are a number of limitations to this study. The most important limitation is the concern that the sample size may not be large enough or the sample may not be adequately representative for the results to be reliable. First, there is a potential selection bias (i.e., respondents may have interest in TP). The final sample is small, compared with the total number of psychiatry trainees nationally (i.e., approximately 225 residency programs with 6700 residents and 1200 fellows/ year, including 900 fellows in the 140 child and adolescent psychiatry programs; $51+\%$ of residents are female with 7\% unreported) (American Psychiatric Association, 2019). Time, high volume email, and other interests were constraints in folks volunteering. We did not send out a survey to a specific group and did not have resources to target an entire organization. In retrospect, it may have been better to secure commitments from 10 programs, get co-investigators, go through the IRBs more formally. Second, a yes/no survey design is not as discerning as a Likert-type design. Its reliability/validity was not studied. Third, the survey did not technically define experience or exposure, so it could have been interpreted as clinical or didactic. Fourth, respondents' self-identified information that was not confirmed in terms of how they defined or rated their clinical experience - nor was it temporally connected to it - though the survey was looking at ranges of experience rather than specific quantitative data. Fifth, statistically significant correlations do not indicate causation. Sixth, more specific demographic and training data would be helpful (e.g., year of training, year when exposed to TP). Finally, there is no "gold standard" for norms by which to compare the respondents' data (i.e., normal anxiety about internet disconnections).

Despite the limitations, the current study provides valuable preliminary data about telepsychiatric use, interest, and attitudes that may serve as a foundation for further research and training.

\section{Conclusions}

Overall, telepsychiatry is effective for adult and child and adolescent populations. Interest in TP is substantial and growing among patients and trainees, which will facilitate its use for accessible, timely and high-quality care. Child psychiatrists are embracing technology and appear to be a leading force in its adoption and implementation. Training, experience, and supervision will reduce concerns and improve confidence. Ongoing research on the use of TP for psychiatric care, the sharing of curricular resources and teaching expertise among programs, and a focus on core residency training competencies will improve positive outcomes, including learner outcomes of skill, knowledge, and attitudes. Residency and fellowship training appears to be an ideal time for an integrated and inter-institutional TP curriculum. This is feasible with the support of academic and healthcare institutions, national professional organizations, and research funding.

\section{References}

Academy, \& of Child and Adolescent Psychiatry (AACAP) Committee on Telepsychiatry and AACAP Committee on Quality Issues. (2017). Clinical update: telepsychiatry with children and adolescents. Journal of the American Academy of Child and Adolescent Psychiatry, 56(10), 875-893.

American Academy of Child and Adolescent Psychiatry-American Psychiatric Association. (2019). Child and Adolescent Telepsychiatry Toolkit. Retrieved from: https://www.psychiatry. org/psychiatrists/practice/telepsychiatry/toolkit/child-adolescent.

American Psychiatric Association. (2019). 2018 Resident/ Fellow Census. file:///U:/Telemed/Key\%20Docs\%20\&\%20Pubs/APAResident-Census-2019.pdf.

American Psychiatric Association and American Telemedicine Association. (2018). Best Practices in Videoconferencing-Based Telemental Health. Retrieved from: https://www.psychiatry.org/psychiatrists/ practice/telepsychiatry/blog/apa-and-ata-release-new-telementalhealth-guide.

American Telemedicine Association. Practice guideline for child and adolescent telemental health. (2017). Telemedicine Journal and E-Health. Retrieved from: https://higherlogicdownload.s3. amazonaws.com/AMERICANTELEMED/618da447-dee1-4ee1b941-c5bf3db5669a/UploadedImages/Practice\%20Guideline\% 20Covers/NEW_ATA\%20Children\%20\&\%20Adolescents\% 20Guidelines.pdf.

Armstrong, E. G., Mackey, M., \& Spear, S. J. (2004). Medical education as a process management problem. Academic Medicine, 79(8), 721-728. https://doi.org/10.1097/00001888-200408000-00002.

Balon, R., Beresin, E. V., Coverdale, J. H., Louie, A. K., \& Roberts, L. W. (2015). Strengthening telepsychiatry's role in clinical care and education. Academic Psychiatry, 39(1), 6-9. https://doi. org/10.1007/s40596-014-0265-x.

Crawford, A., Sunderji, N., López, J., \& Soklaridis, S. (2016). Defining competencies for the practice of telepsychiatry through an assessment of resident learning needs. BMC Medical Education, 16, 28. https://doi.org/10.1186/s12909-016-0529-0.

Cruz, C., Orchard, K., Shoemaker, E. Z., Hilty, D. M. (2020). A survey of residents/fellows, program directors and faculty about telepsychiatry: clinical experience, interest, views and misconceptions, Journal of Technology in Behavioral Science, In Press

DeJong, S. M. (2018). Professionalism and technology: competencies across the tele-behavioral health and e-behavioral health spectrum. Academic Psychiatry, 42(6), 800-807. https://doi. org/10.1007/s40596-018-0947-x. 
Downey, R. G., \& King, C. (1998). Missing data in Likert ratings: a comparison of replacement methods. The Journal of General Psychology, 125(2), 175-191. https://doi.org/10.1080/00221309809595542.

Gloff, N. E., LeNoue, S. R., Novins, D. K., \& Myers, K. (2015). Telemental health for children and adolescents. International Review of Psychiatry (Abingdon, England), 27(6), 513-524. https://doi. org/10.3109/09540261.2015.1086322.

Glover, J. A., Williams, E., Hazlett, L. J., \& Campbell, N. (2013). Connecting to the future: telepsychiatry in postgraduate medical education. Telemedicine Journal and E-Health, 19(6), 474-479. https://doi.org/10.1089/tmj.2012.0182.

Hilty, D. M., Chan, S., Torous, J., Luo, J., Boland, R. J. (2020). A framework for competencies for the use of mobile technologies in psychiatry and medicine. Journal of Medical Internet Research Uhealth and Mobile Health, 2020; 8(2) http://mhealth.jmir. org/2020/2/e12229/.

Hilty, D. M., Crawford, A., Teshima, J., Chan, S., Sunderji, N., Yellowlees, P. M., \& Li, S. T. (2015). A framework for telepsychiatric training and e-health: competency-based education, evaluation and implications. International Review of Psychiatry, 27(6), 569-592. https://doi.org/10.3109/09540261.2015.1091292.

Hilty, D. M., Feliberti, J., Evangelatos, G., Lu, F. G., \& Lim, R. F. (2018). Competent cultural telebehavioral healthcare to diverse populations: administration, evaluation, and financing. Journal of Technology in Behavioral Science, 4(3), 186-200. https://doi. org/10.1007/s41347-018-0076-0.

Hilty, D. M., Ferrer, D. C., Parish, M. B., Johnston, B., Callahan, E. J., \& Yellowlees, P. M. (2013). The effectiveness of telemental health: a 2013 review. Telemedicine Journal and E-Health, 19(6), 444-454. https://doi.org/10.1089/tmj.2013.0075.

Hilty, D. M., Gentry, M. T., McKean, A. J., Cowan, K. E., Lim, R. F., \& Lu, F. G. (2020). Telehealth for rural diverse populations: telebehavioral and cultural competencies, clinical outcomes and administrative approaches. mHealth, 6, 20. https://doi. org/10.21037/mhealth.2019.10.04.

Hilty, D. M., Maheu, M. M., Drude, K. P., \& Hertlein, K. M. (2018). The need to implement and evaluate telehealth competency frameworks to ensure quality care across behavioral health professions. Academic Psychiatry, 42(6), 818-824. https://doi. org/10.1007/s40596-018-0992-5.

Hilty, D. M., Randhawa, K., Maheu, M. M., McKean, A. S., Pantera, R., \& Rizzo, A. (2020). A review of telepresence, virtual reality and augmented reality applied to clinical care. Journal of Technology in Behavioral Science. https://doi.org/10.1007/s41347-020-00126-x.

Hilty, D. M., Shoemaker, E. Z., Myers, K., Snowdy, C. E., Yellowlees, P. M., \& Yager, J. (2016). Need for and steps toward a clinical guideline for the telemental healthcare of children and adolescents. Journal of Child and Adolescent Psychopharmacology, 26(3), 283-295. https://doi.org/10.1089/cap.2015.0129.

Hilty, D. M., Sunderji, N., Suo, S., Chan, S., \& McCarron, R. M. (2018). Telepsychiatry and other technologies for integrated care: evidence base, best practice models and competencies. International Review of Psychiatry (Abingdon, England), 30(6), 292-309. https://doi.org/10.1080/09540261.2019.1571483.

Hilty, D. M., Torous, J., Parish, M., Chan, S., Xiong, G., Scher, L., \& Yellowlees, P. M. (2020). A literature review comparing clinicians' approaches and skills to in-person, synchronous and asynchronous care: moving toward asynchronous competencies to ensure quality care. Telemedicine Journal and E-Health, 2020;https://doi.org/10.1089/tmj.2020.0054.

Hilty, D. M., Unützer, J., Ko, D. G., Luo, J., Worley, L., \& Yager, J. (2019). Approaches for departments, schools, and health systems to better implement technologies used for clinical care and education. Academic Psychiatry, 43(6), 611-616. https://doi. org/10.1007/s40596-019-01074-2.
Hoffman, P., \& Kane, J. M. (2015). Telepsychiatry education and curriculum development in residency training. Academic Psychiatry, 39(1), 108-109. https://doi.org/10.1007/s40596-013-0006-6.

Horvath, A. O., \& Greenberg, L. S. (1989). The development and validation of the Working Horvath, A. O., Greenberg, L. S. (1989). The development and validation of the Working Alliance Inventory. Journal of Counseling Psychology, 36, 223-233. https://doi. org/10.1037/0022-0167.36.2.223.

Hubley, S., Lynch, S. B., Schneck, C., Thomas, M., \& Shore, J. (2016). Review of key telepsychiatry outcomes. World Journal of Psychiatry, 6(2), 269-282. https://doi.org/10.5498/wjp.v6.i2.269.

Joshi, S. V., Stubbe, D., Li, S. T., \& Hilty, D. M. (2019). Correction to: The use of technology by youth: implications for psychiatric educators. Academic psychiatry: the journal of the American Association of Directors of Psychiatric Residency Training and the Association for Academic Psychiatry, 43(4), 466. https://doi. org/10.1007/s40596-018-1013-4.

Khan, S., \& Ramtekkar, U. (2019). Child and adolescent telepsychiatry education and training. The Psychiatric Clinics of North America, 42(4), 555-562. https://doi.org/10.1016/j.psc.2019.08.010.

Krishna, R. (2017). The impact of health information technology on the doctor-patient relationship in child and adolescent psychiatry. Child and Adolescent Psychiatric Clinics of North America, 26(1), 67-75. https://doi.org/10.1016/j.chc.2016.07.007.

Levy, S., \& Strachan, N. (2013). Child and adolescent mental health service providers' perceptions of using telehealth. Mental Health Practice, 17(1), 28-32. https://doi.org/10.7748/mhp2013. 09.17.1.28.e810.

Maheu M., Drude, K., Hertlein, K. M., Hilty, D. M. (2018). A framework for interprofessional telebehavioral health competencies: implementation and challenges moving forward. Academic Psychiatry, 42(6), 825-833. https://doi.org/10.1007/ s40596-018-0988-1. Epub 2018 Oct 3.

Maheu M., Drude, K., Hertlein, K., Lipschutz, R., Wall, K., Long, R., et al., \& Hilty, D. M. (2019). An interdisciplinary framework for telebehavioral health competencies. Journal of Technology in Behavioral Science, 3(2), 108-40; correction 3(2):107. https://doi. org/https://doi.org/10.1007/s41347-019-00113-

Myers, K., Vander Stoep, A., Zhou, C., McCarty, C. A., \& Katon, W. (2015). Effectiveness of a telehealth service delivery model for treating attention-deficit/hyperactivity disorder: a communitybased randomized controlled trial. Journal of the American Academy of Child and Adolescent Psychiatry, 54(4), 263-274. https://doi.org/10.1016/j.jaac.2015.01.009.

Nelson, E. L., \& Sharp, S. (2016). A review of pediatric telemental health. Pediatric Clinics of North America, 63(5), 913-931. https:// doi.org/10.1016/j.pcl.2016.06.011.

Nelson, E. L., Cain, S., \& Sharp, S. (2017). Considerations for conducting telemental health with children and adolescents. Child and Adolescent Psychiatric Clinics of North America, 26(1), 77-91. https://doi.org/10.1016/j.chc.2016.07.008.

Pakyurek, M., Yellowlees, P., \& Hilty, D. (2010). The child and adolescent telepsychiatry consultation: can it be a more effective clinical process for certain patients than conventional practice? Telemedicine Journal and E-health, 16(3), 289-292. https://doi. org/10.1089/tmj.2009.0130.

Odgers, C. L., \& Jensen, M. R. (2020). Annual research review: adolescent mental health in the digital age: facts, fears, and future directions. Journal of Child Psychology and Psychiatry, and Allied Disciplines, 61(3), 336-348. https://doi.org/10.1111/jcpp.13190.

Russell, M. A., \& Gajos, J. M. (2020). Annual research review: ecological momentary assessment studies in child psychology and psychiatry. Journal of Child Psychology and Psychiatry, and Allied Disciplines, 61(3), 376-394. https://doi.org/10.1111/ jcpp.13204. 
Pratt, D. D. (1998). Five perspectives on teaching in adult and higher education. Malabar, FL: Krieger Publishing.

Reliford, A., \& Adebanjo, B. (2019). Use of telepsychiatry in pediatric emergency room to decrease length of stay for psychiatric patients, improve resident on-call burden, and reduce factors related to physician burnout. Telemedicine Journal and E-Health, 25(9), 828-832. https://doi.org/10.1089/tmj.2018.0124.

Robillard, G., \& Bouchard, S. (2004). The sense of presence in videoconferencing and emotional engagement. Cyberpsychology and Behavior, 7(3), 280-281.

Schneider, P. L. (1999). Mediators of distance communication technologies psychotherapy: development of a measure. Paper presented to the American Psychological Association, Boston. Retrieved from: https:// www.studio5d.com/paul/research/DCCS.html

Stiles, W. B., Reynolds, S., Hardy, G. E., Rees, A., Barkham, M., \& Shapiro, D. A. (1994). Evaluation and description of psychotherapy sessions by clients using the Session Evaluation Questionnaire and the Session Impacts Scale. Journal of Counseling Psychology, 41(2), 175-185. https://doi.org/10.1037/0022-0167.41.2.175.

Sunderji, N., Crawford, A., \& Jovanovic, M. (2015). Telepsychiatry in graduate medical education: a narrative review. Academic Psychiatry, 39(1), 55-62. https://doi.org/10.1007/s40596-014-0176-x.

Teshima, J., Hodgins, M., Boydell, K. M., \& Pignatiello, A. (2016). Resident evaluation of a required telepsychiatry clinical experience. Academic psychiatry: the journal of the American Association of Directors of Psychiatric Residency Training and the Association for Academic Psychiatry, 40(2), 348-352. https:// doi.org/10.1007/s40596-015-0373-2.
Torous, J., Jän Myrick, K., Rauseo-Ricupero, N., \& Firth, J. (2020). Digital mental health and covid-19: using technology today to accelerate the curve on access and quality tomorrow. Journal of Medical Informatics Research Mental Health, 7(3), e18848. https:// doi.org/10.2196/18848.

Wang, Q. E., Myers, M. D., Sundaram, D. (2013). Digital natives and digital immigrants towards a model of digital fluency. Busin Inform Syst Engin, 5(6), 409-419. https://doi.org/10.1007/s11576-013-0390-2.

Yip, M. P., Chang, A. M., Chan, J., \& MacKenzie, A. E. (2003). Development of the Telemedicine Satisfaction Questionnaire to evaluate patient satisfaction with telemedicine: a preliminary study. Journal of Telemedicine and Telecare, 9(1), 46-50. https:// doi.org/10.1258/135763303321159693.

Zalpuri, I., Liu, H., Stubbe, D., Wrzosek, M., Sadhu, J., \& Hilty, D. (2018). A competency-based framework for social media for trainees, faculty and others. Academic Psychiatry, 42(6), 808-817. https://doi.org/10.1007/s40596-018-0983-6.

Publisher's Note Springer Nature remains neutral with regard to jurisdictional claims in published maps and institutional affiliations. 